Diogo Barth Pacini ${ }^{1}$

Carla Regina de Mendonça dos Santos ${ }^{2}$ Camilla Rocha Teixeira ${ }^{3}$ Paulo Roberto Martins Queiroz ${ }^{4}$
* Recebido em: 04/05/2014.

Aprovado em: 14/05/2015.

1 Esp. Biotecnologia. Graduado em Biologia. UniCEUB. E-mail: diogo.pacini@gmail.com.

2 Esp. Biociências Forense. Graduanda do curso de Biomedicina. UniCEUB. E-mail: crmendonca@gmail.com.

3 Curso de biomedicina da Faculdade de Ciências da Educação e Saúde - FACES. Centro Universitário de Brasília.

$4 \mathrm{PhD}$ em Biologia Animal. Universidade de Brasília - UnB. Professor do curso de Biomedicina. UniCEUB.E-mail: pqsilva@uol.com.br.

\section{Uso dos diptera na análise entomotoxicologica e na estimativa do intervalo pós-morte (IPM)*}

\section{Use of diptera in entomotoxicological analysis and determination of the post mortem interval (PMI)}

\section{Resumo}

A entomologia forense consiste no estudo de insetos que são encontrados em locais de crimes, e são utilizados como vestígios, aumentando a possibilidade de encontrar a causa da morte e a estimar o intervalo pós-morte (IPM). Com base nessas análises, os entomólogos e os peritos observaram que os componentes químicos utilizados pelos indivíduos, tais como medicamentos ou drogas, ou até mesmo o uso de venenos, alteravam o que já se conhecia da estimativa do IPM, surgindo, desse modo, nova vertente nessa ciência, que é a entomotoxicologia forense. O presente trabalho bibliográfico descreve os princípios básicos da entomotoxicologia abordando principalmente a interferência na estimativa do intervalo pós-morte.

Palavras-chave: Entomologia forense. Entomotoxicologia. Díptera. Inseto. IPM. Tempo de morte.

\begin{abstract}
Forensic entomology is the study of insects that are found at crime scenes, and are used as traces, increasing the possibility of finding the cause of death and to estimate the postmortem interval (PMI). Based on these analyzes entomologists and the experts noted that the chemical components used by the people, such as medications or drugs, even the use of poison changed what was already known of estimating the PMI, with this, emerge a new dimension in this science, which is the forensic entomotoxicology. This bibliographic study describes the basic principles of entomotoxicology mainly addressing interference to estimate the postmortem interval.
\end{abstract} Keywords: Forensic entomology. Entomotoxicology. Diptera. Insect. PMI. Time of death. 


\section{Introdução}

A entomologia consiste no estudo dos Arthropoda, um dos maiores e mais diversificados grupos do Reino Animal, compreendendo os insetos, crustáceos, aracnídeos, miriápodes, entre outros. Surgidos de organismos vermiformes, os artrópodes, em sua evolução, com base em processo de tagmatização, deram origem a superclasse Hexapoda que reúne organismos cujo corpo se divide em três diferentes regiões: cabeça, tórax e abdome. Em 1981, Hennig, a fim de tornar independentes as classes Collembola, Protura, Diplura e Insecta, propôs juntá-las dando-lhes o nome de Hexapoda (OLIVEIRA-COSTA, 2003).

Entre as infraclasses da classe Insecta, encontram-se os insetos da classe Neoptera que deram origem a ordem Diptera, em que estão inseridas as famílias mais importantes para a Entomologia Forense (GOFF, 2001; GENNARD, 2007), tais como, Calliphoridae (mosca varejeira ou mosca metálica), Sarcophagidae (mosca da carne ou de pijama) e Muscidae (mosca doméstica) são as primeiras espécies a colonizarem os cadáveres. Além dessas, famílias como Piophilidae (mosca do queijo) e Sepsidae (mosca necrófaga negra) de ordem também Diptera chegam mais tarde para a colonização do cadáver (GENNARD, 2007).

Um dos primeiros sinais após a morte que auxilia na colonização do cadáver é a decomposição química das células, dando início a liberação de enzimas e a atividade das bactérias e fungos que começam pelo intestino e ambiente externo, devido às mudanças físico-químicas causadas em corpos humanos e de outros animais (AMENDT et al., 2004; GENNARD, 2007), gerando inchaços ao longo do corpo e odores atraindo os primeiros organismos da família Calliphoridae até o local (WALL; WARNES, 1994; ANDERSON, 2001; GENNARD, 2007). Os insetos atraídos pelo corpo demoram poucos minutos para chegar à área (ERZINCLIOGLU, 1983; SMITH, 1986; ANDERSON, 2001).

A toxicologia tem sua origem etimológica proveniente do grego "Toxikon", podendo ser definida como o estudo dos agentes tóxicos que interagem com os sistemas vivos por meio de processos químicos (KLAASSEN et al., 1996). A toxicologia forense tem como principal objetivo a detecção e quantificação de substâncias tóxicas eventualmente presentes em situações criminais (ALVES, 2005).
O uso da entomotoxicologia é de interesse relevante devido à determinação da droga utilizada pelo indivíduo pouco antes da morte, sendo aplicado em especial em restos esqueletizados nos quais não há presença de tecido ou fluido no local (GOSSELIN et al., 2011). Entomologistas, a fim de tornar essa ferramenta útil para fins forenses, começaram a detectar drogas em insetos a partir de 1980 (BEYER et al., 1980; INTRONA et al., 2001). Kintz et al (1990) analisaram e obtiveram resultados de que as concentrações de drogas poderiam ser mais estáveis no inseto do que no cadáver, o que ajudaria pelo fato de pupários e exúvias estarem presentes por mais tempo quando já não seria mais possível encontrar amostras toxicológicas disponíveis (BOUREL et al., 1999).

Uma das ferramentas mais utilizadas para maior precisão na investigação do tempo de decomposição do cadáver é o intervalo pós-morte (IPM). O uso do IPM tem aplicações na área cível, casos de morte natural, acidental ou suicídio (GREENBERG; KUNICH, 2002), ele é importante na reconstrução da cena do crime quando há suspeitos, podendo também auxiliar em melhor análise das informações fornecidas por testemunhas (PINHEIRO et al., 2012), como também, na determinação do intervalo do tempo de morte (IPM).

O IPM refere-se ao tempo entre a morte e a descoberta do corpo (CATTS, 1992; AMENDT et al., 2011) com a ajuda de evidências entomológicas, principalmente larvas de moscas varejeiras de ordem Diptera, consideradas as melhores para as determinações dessa estimativa (CENTEIO, 2011). Aproximadamente existem cinquenta espécies dessas moscas identificadas como potenciais indicadoras do IPM (GRUNER et al., 2007). A estimativa por tais meios depende do inseto e estágio de desenvolvimento, que este apresenta, quando coletados já no corpo em decomposição (VOSS et al., 2011).

Por meio deste estudo buscou-se descrever os princípios básicos da entomotoxicologia forense na estimativa do IPM.

\section{Metodologia}

Trata-se de uma revisão bibliográfica narrativa baseada em trabalhos científicos que trataram do tema em questão. Parte do material utilizado foi obtida na internet, em sítios de pesquisa como: Scientific Electronic Library Online (SciELO), Google Acadêmico, Repositório Institucional da UnB e de outras Universidades, entre 
outras bases de dados. Outra parte do material utilizado foi obtida por meio de teses e monografias de mestrado e doutorado.

\section{Entomologia forense}

Uma das primeiras e mais importantes aplicações da entomologia forense na história aconteceu no século XIII, quando Sung Tz’u, um investigador chinês, ao buscar vestígios de um assassinato ocorrido na aldeia, observou a ocorrência de insetos sobre uma das foices dos suspeitos depositadas ao solo, devido aos resquícios de sangue, levando o proprietário a confessar o crime (SUNG; MCKNIGHT, 1981). Com isso, novos estudos foram surgindo a respeito dos insetos, como no caso do pesquisador Orfila (1848), talvez o primeiro patologista a sistematizar a sucessão de 30 insetos e artrópodes que se alimentaram e colonizaram um cadáver (GREENBERG; KUNICH, 2002). Outro caso constitui a primeira utilização dessa ciência com insetos como indicadores forenses por Bergeret (1855), ao descobrir o corpo de uma criança embaixo de um piso oculto de uma residência. Mas foi com a publicação do livro: "La faune dês cadavres" por Mégnin (1894), que os dados entomológicos ficaram mundialmente conhecidos como uma nova ajuda nas investigações forenses, estabelecendo uma nova ciência (GREENBERG; KUNICH, 2002; OLIVEIRA-COSTA, 2003; KELLY, 2006).

A entomologia forense é uma ciência que consiste no uso de artrópodes como indicadores biológicos a procedimentos legais, sendo uma ferramenta de auxílio para a determinação na estimativa do intervalo pós-morte (IPM) em investigações policiais (OLIVEIRA-COSTA, 2003; KELLY, 2006; PINHEIRO et al., 2012). É possível com base em estudos, tais como, o de Lord (1990), Benecke e Leggig (2001), Campobasso e Introna (2001); Carvalho et al., (2004), utilizar a entomologia forense, a fim de descobrir o tempo desde a morte, local geográfico da morte, se houve ou não movimentação do cadáver, estação meteorológica do óbito, tempo de desmembramento, intervalo de submersão, uso de tóxicos ou drogas, as lesões no corpo, negligência em crianças, abuso sexual, identificação ou ligação do suspeito à cena de crime, entre outros. Podendo também ser aplicada em casos de caça ilegal e maus tratos (ANDERSON, 1999; WATSON; CARLTON, 2003; WATSON; CARLTON, 2005).
$\mathrm{O}$ uso da entomologia forense pode ser dividido em três áreas distintas: urbana, na qual os insetos são encontrados em ambiente habitável, causando danos ao homem; de produtos acumulados, deixando grande quantidade de alimento contaminado; e médico-legal, envolvendo a área criminal, principalmente, relacionado à morte, lidando principalmente com artrópodes (LORD; STEVENSON, 1986).

Existem três estádios larvares depois que os ovos são eclodidos (CHARABIZDE et al., 2009; GOSSELIN et al., 2010), seguidos de um pupal e a fase adulta. Após o terceiro estádio, quando a larva atinge o seu tamanho máximo, ela deixa de se alimentar e procura um lugar para se tornar uma pupa, longe da sua fonte de alimento (GOSSELIN et al., 2011; PARRY et al., 2011). Essa fase é, na maioria das vezes, a mais representada em amostra de insetos forenses (GOSSELIN et al., 2010). Cada fase possui uma duração que, devido a fatores bióticos e abióticos, pode ser afetada (PARRY et al., 2011).

$\mathrm{O}$ desenvolvimento do inseto pode ser afetado quando sua fonte de alimento possui algum tipo de droga envolvida. Cada tipo de droga possui um efeito em cada espécie e, dependendo da concentração e do metabolismo dentro do inseto, um fármaco pode fazer com que possa ocorrer uma aceleração ou regressão durante suas fases. A quantidade a qual o inseto pode absorver, excretar e fixar da droga dentro de seu metabolismo influencia na concentração interna desses. A excreção de droga pelos insetos, em muitos casos, é muito eficaz, tornando a concentração da droga no interior da larva em níveis mais baixos do que a fonte de alimento (PARRY et al., 2011).

\section{Entomotoxicologia}

A entomotoxicologia é uma área recente na entomologia forense que utiliza insetos necrófagos com o propósito de identificar e determinar, qualitativamente e quantitativamente, substâncias tóxicas, tais como, drogas ou toxinas presentes no cadáver e investigar os possíveis efeitos que podem causar no desenvolvimento desses insetos (INTRONA et al., 2001; GAGLIANO-CANDELA; AVENTAGGIATO, 2001), os quais interferem nos fatores usados para a estimativa do IPM. O entendimento da dinâmica dos artrópodes colonizando o cadáver pode auxiliar os peritos na determinação de quais toxinas estavam presentes no corpo no momento 
da morte (GAGLIANO-CANDELA; AVENTAGGIATO, 2001). Um conhecimento do efeito de tais substâncias tóxicas é necessário para melhor entendimento de como isso pode influenciar no comportamento do inseto sobre o cadáver (SOTO, 2008).

O uso dos insetos como ferramenta para detecção de substâncias toxicológicas ocorreu pela primeira vez por Nuorteva (1977), que conseguiu detectar concentrações baixas de mercúrio por meio de larvas que se alimentavam do corpo, sendo possível encontrar a origem geográfica deste (GANGLIANO-CANDELA; AVENTAGGIATO, 2001; GOFF; LORD, 2001). No entanto, o primeiro estudo de caso usando insetos na área forense foi feito por Beyer et al. (1980). Um corpo de uma mulher de 22 anos foi encontrado após 14 dias em estado avançado de decomposição, sem que houvesse qualquer material biológico para a análise toxicológica. No local foi encontrado um frasco vazio de fenobarbital (anticonvulsivante) com capacidade de 100 comprimidos. Larvas da mosca Cochliomyia macellaria foram coletadas no local e utilizadas para detecção por cromatografia gasosa e confirmada por cromatografia de camada fina. Essa foi a primeira identificação feita pelo auxílio de insetos, tornando, assim, a entomotoxicologia estabelecida.

Com a determinação qualitativa, pode-se obter informações como a causa da morte até a área geográfica onde foi encontrado o corpo (NUORTEVA, 1977). O uso da entomotoxicologia é aplicado na área forense de forma cada vez mais frequente. O número de casos de morte relacionado a drogas ou a algum tipo de intoxicação sendo ela acidental ou suicida, tem aumentado a sua demanda (INTRONA et al., 2001; MURTHY; MOHANTY, 2010). A identificação dessas possíveis drogas é feita com ajuda de insetos, principalmente os da ordem Díptera, por serem os primeiros a colonizar o cadáver (INTRONA et al., 2001).

Em muitas situações, não é possível adquirir amostras confiáveis quando o corpo se apresenta em estado esqueletizado ou em estado avançado de decomposição, não podendo ter acesso a sangue, urina ou órgãos internos. Nessas situações os insetos são usados como material, sendo homogeneizados e submetidos a procedimentos comuns de análises toxicológicas, como radioimunoensaio (RIA), cromatografia gasosa (CG), cromatografia gasosa com espectrometria de massas (GC/ MS), cromatografia em camada fina (TLC), cromatogra- fia líquida de alta pressão (HLPC) e imunohistoquímica (IHQ) (INTRONA, 2001; PAYNE-JAME et al., 2005). Ao se alimentarem do corpo em decomposição, esses invertebrados podem acumular drogas ou tóxicos ingeridos pelo indivíduo antes do óbito (GOFF; LORD, 2001; GENNARD, 2007).

Espécies de insetos necrófagos da ordem Diptera e/ou Coleoptera são os mais recomendados para estudos entomotoxicológicos, por serem os primeiros a colonizar o corpo (AMENDT et al., 2006; DEFINIS-GOJANOVI'C et al., 2007), pois, ao se alimentarem do cadáver em decomposição, eles podem acumular substâncias consumidas pela pessoa antes da morte (GOFF; LORD, 2001; GENNARD, 2007). Uma parte desses insetos bioacumulam drogas ou outros tóxicos, cômoda mesma forma que, parasitóides, predadores ou espécies onívoras. A bioacumulação não acontece de forma igual para todos, já que ela difere devido ao comportamento durante a alimentação ou seu ciclo de vida (NUORTEVA, 1977; MILLER et al., 1994; FARIA et al., 1999).

A estabilidade do fármaco nos tecidos do inseto é influenciado por vários agentes ambientais, tais como, a temperatura, umidade e radiação UV que influenciam na estabilidade do fármaco nos tecidos do inseto (TRACQUI et al., 2004), assim como sua absorção, excreção e a motilidade do intestino, além do seu desenvolvimento (PARRY et al., 2011) podendo, esses fatores, causar interferência nos níveis de concentração da droga (GOSSELIN et al., 2011).

A amostragem de insetos, variada entre pupas e larvas, constitui fator de elevada mobilidade na detecção das drogas (GOSSELIN et al., 2011). Diversos autores (SADLER et al., 1995; TRACQUI et al., 2004; CARVALHO, 2010), em estudos variados, descobriram que a coleta em diferentes lugares do corpo era muito importante, devido às mudanças de concentrações por causa das propriedades físico-químicas do fármaco, quando esse entra em contato com órgão e tecidos diferentes, logo, alterando a concentração nos insetos. Muitos preferem amostras aleatórias, tais como, órgãos internos como fígado (na maior parte das vezes), cabeça e músculos (INTRONA et al., 1990; DEFINIS-GOJANOVIC et al., 2007) e na superfície da pele (FARIA et al., 1999). Amendt et al. (2006) e Carvalho (2010), publicaram algumas normas e um protocolo para amostragem no campo da entomotoxicologia (Figura 1). 
Figura 1 - Folha de protocolo de análise de amostras entomotoxicológicas. Os códigos indicam: L, liver (fígado); B, brain (cérebro); $M$, muscle (músculo); $O$, others (outros órgãos). $\left({ }^{* *}\right)$ L, larvae (larva); P, pupae (pupa); EP, empty pupae (pupa vazia); A, adults (adultos).

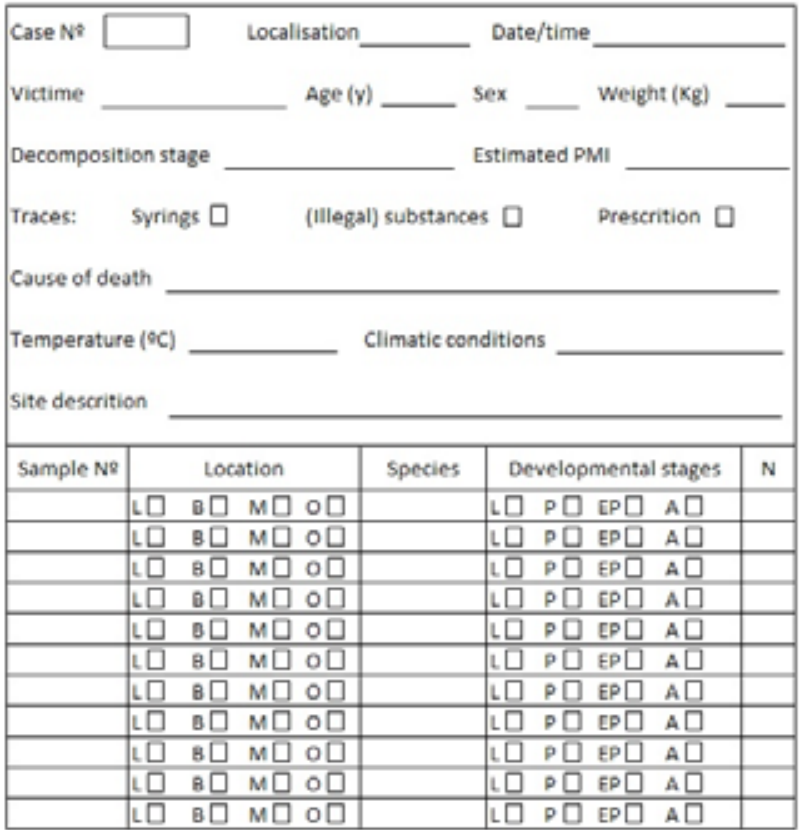

Fonte: (AMENDT et al., 2006; CARVALHO, 2010).

Wyman et al. (2011), ao monitorar vários tipos de drogas em tecidos de suínos em decomposição, observaram que, conforme o estado de decomposição evoluía, a concentração das drogas aumentava, havendo diferença de concentração entre elas. Também relataram concentrações muito maiores no fígado desses suínos do que nos músculos. Assim, esse estudo, além de indicar o aumento das concentrações, revela que quanto maior o tempo que o inseto estiver exposto ao cadáver maior será o efeito sobre seu desenvolvimento, sendo um fator a mais na amostragem.

Campobasso et al. (2004), ao realizarem um estudo com 18 casos, analisaram drogas de larvas e tecidos humanos. Com opiáceos, observaram que, em quatro casos, a concentração da droga em larvas de alimentação ativa era mais elevada do que em amostras de sangue e que larvas de pós-alimentação apresentaram redução significativa de concentração, devido ao fato da taxa de eliminação ser maior do que a taxa de absorção. Em outros quatro, as concentrações de cocaína eram iguais tanto em amostras de larvas de alimentação quanto em amostras de fígado. A concentração de cocaína em amostra de fígado era muito maior do que para amostras de sangue e amostras de larvas pós-alimentação, com exceção de três casos. Nos casos com antidepressivos, como levomepromazina, amitriptili- na, a concentração era maior em larvas de alimentação do que em amostras de sangue, porém maior em amostras de fígado. Somente clomipramina foi encontrada na mesma concentração para todas as amostras. Em casos de concentração com fenobarbital, observou-se maior nível para larvas pós-alimentação do que para larvas de alimentação. No entanto, ainda sendo nível menor do que a concentração em amostras de sangue e fígado.

Quando feita a coleta das espécies no local do crime, é preciso realizar procedimento adequado para a preservação do espécime. Ao serem removidos do corpo, eles são lavados com água da torneira ou água destilada e armazenadas a uma temperatura que varia de -20 ${ }^{\circ} \mathrm{C}$ a $4{ }^{\circ} \mathrm{C}$. Após essa etapa, eles serão lavados novamente removendo os fluidos do cadáver e então seguindo aos procedimentos laboratoriais (GAGLIANO-CANDELA; AVENTAGGIATO, 2001). Dependendo da suspeita da droga, a preparação dessas amostras pode diferir em inúmeras formas (MURTHY; MOHANTY, 2010; GOSSELIN et al., 2011). Caso essas amostras sejam sólidas, terão que ser maceradas e homogeneizadas (WOLFF et al., 2004; GUNN et al., 2006), podendo também ser digeridas com ácidos, bases ou enzimas para quebrar o exoesqueleto quitinoso e liberar possíveis toxinas (ROETERDINK et al., 2004; GAGLIANO-CANDELA; AVENTAGGIATO, 2001) ou ainda, por pulverização, sendo possível evitar contaminação cruzada entre amostras diferentes (GOSSELIN et al., 2010). Com a pulverização das amostras, as drogas serão extraídas pela matriz, porém ainda técnicas tais como precipitação de proteína, extração líquido-líquido (ELL) ou extração em fase sólida (SPE) ainda são as mais utilizadas pelos entomotoxicologistas (GOSSELIN et al., 2011).

Como já dito, drogas ou tóxicos podem influenciar no crescimento e desenvolvimento de um inseto podendo mudar a determinação mais precisa do IPM, por isso experimentos de laboratório são realizados para saber quais são os possíveis efeitos (DACKO, 2011).

Soto (2008) ao desenvolver pesquisa com larvas de três tipos de moscas diferentes, Chrysomya megacephala, Chrysomya putoria e Chrysomya albiceps, utilizando fenobarbital em três dosagens diferentes em suas dietas, observou que o desenvolvimento dessas larvas eram similiares ao controle quando estavam em dosagens menores, porém, ao aumentar as dosagens, o desenvolvimento se apresentava muito mais lento do que as larvas do grupo controle. 
Ao utilizar as mesmas três espécies do experimento anterior, Lima (2009) observou os efeitos do ectasy no desenvolvimento dessas moscas. Dentre as três espécies, apenas Chrysomya megacephala apresentou diferença em relação ao tempo de maturação quando comparadas ao grupo controle, enquanto C. putoria e C. albiceps não demonstraram variações significativas.

Em outro estudo, Mahat et al. (2009), ao analisar larvas e pupas de Chrysomya megacephala na presença de malathion, observaram retardo no tempo de desenvolvimento, indicando que a droga pode influenciar ao estimar o índice pós-morte (IPM) após seu uso.

\section{Intervalo pós-morte (IPM)}

O uso dos insetos consiste no principal meio para uma base do IPM, pois são os primeiros a chegar ao local do crime minutos após o óbito, mesmo a longas distâncias (CATTS; GOFF, 1992; ERZINCLIOGLU, 1996; ANDERSON, 2001). O corpo humano, após a morte, passa por vários processos de mudança provocados por fungos, bactérias e decomposição química liberando enzimas e gases para o meio externo, iniciando uma das fases de decomposição do cadáver (AMENDT et al., 2004). A chegada dos insetos é dividida em ondas diferentes dependendo da fase de decomposição em que o corpo se encontra, podendo também ser influenciada por temperaturas, profundidades (em caso de sepultamento), ou quando este se apresenta embalado ou coberto (BACHMANN; SIMMONS, 2010).

O IPM é estimado com base em métodos entomológicos baseados na sucessão dos insetos e na coleta de insetos imaturos no corpo em decomposição para identificar os estágios de desenvolvimento desses (VOSS et al., 2011). É essencial saber se houve oviposição no cadáver pouco depois da morte ou não (AMENDT, 2008). Fatores como chuva, baixas temperaturas, latitude, altitude ou acessibilidade dificultada, pode provocar um retardo na colonização do cadáver pelos insetos (AMENDT, 2004; AMENDT, 2008).

Segundo Lefebvre et al. (2009), o uso de pupas de primeira geração seria melhor do que larvas de segunda geração para estimar a concentração de droga, pois essa é mais baixa em larvas do que em pupários. Entretanto, a quantificação de drogas em larvas pode ser importante para melhor explicação na alteração do desenvolvimento das moscas e, então, para uma estimativa de IPM mais precisa (GOSSELIN et al., 2011a).
$\mathrm{Na}$ entomologia forense, a principal aplicação é a estimativa do intervalo pós-morte (IPM), sendo possível identificar espécies necrófagas encontradas no local, como também, calcular a idade dessas espécies (CATTS, 1992; GREENBERG, 2002; AMENDT, 2004). A presença dos insetos junto ao cadáver é de extrema importância em investigações criminais, sendo possível adquirir informações necessárias para uma melhor estimativa (FERRARI et al., 2008). O IPM está associado ao período de tempo entre a ocorrência do óbito até o momento em que foi encontrado o corpo. Ele tem importância na reconstrução de eventos, na ligação entre o suspeito à cena do crime ou à vítima e na confirmação das informações de terceiros no local (PINHEIRO et al., 2012). A partir de 72h após a morte, a entomologia forense passa a ser a mais precisa e, muitas vezes, a única forma para uma estimativa confiável do IPM (AMENDT, 2011).

Os primeiros colonizadores a chegarem até o corpo são as moscas, da ordem Diptera, de preferência as varejeiras, da família Calliphoridae. Essas são atraídas ao local devido ao odor, podendo localizar o corpo a longas distâncias depositando seus ovos dentro de minutos ou horas após a morte (AMENDT, 2011). A deposição de ovos ocorre em orifícios ou aberturas produzidas por feridas do cadáver. Os decompositores se dividem em dois grupos, os que consomem tecidos moles de carcaças frescas, como é o caso dos Diptera, ou seja, moscas, e os que usam pele e cabelo do cadáver em decomposição para outros fins, em geral são os Coleoptera, como os besouros (PAYNE 1965; PUTMAN, 1983).

Muitas vezes são encontrados apenas ovos das moscas no cadáver, esses ovos podem ser úteis para a estimativa do IPM, por dessecações e análises do estágio do desenvolvimento embrionário (SMITH, 1986; LORD, 1990; ANDERSON, 1999; 2004; BYRD; CASTNER, 2001). Uma forma de identificar espécies pelos ovos encontrados é pela microscopia eletrônica de varredura $(\mathrm{MEV})$. Essa técnica é aplicada em diversos países, porém ainda não é aplicada tão intensivamente no Brasil (SOTO, 2008).

O funcionamento dos insetos sobre e dentro do cadáver acelera o processo de decomposição do cadáver. Esse processo pode ser medido ajudando em uma estimativa mais precisa do IPM, mesmo meses após a morte (CAMPOBASSO et al., 2001). Quando se calcula a idade de desenvolvimento do inseto no corpo, consegue-se também calcular o tempo de colonização que compreen-

\footnotetext{
-
} 
de o IPM mínimo (CATTS, 1992), correspondente ao cálculo que se refere a ocasião em que os insetos chegaram ao corpo e o colonizaram, não incluindo o tempo real da morte (AMENDT et al., 2007).

Devido a todos esses fatores que influenciam no desenvolvimento dos insetos, um processo de três etapas foi formado para proporcionar a entomologia forense um melhor cálculo da idade dos insetos encontrados no local no crime. Essas etapas constituem-se em identificar todas as espécies encontradas no cadáver com precisão, reconstruir as temperaturas conforme à cena do crime e, por final, moldar as taxas de desenvolvimento dos insetos encontradas no corpo (AMENDT et al., 2011).

\section{Considerações Finais}

Atualmente, têm ocorrido muitas mortes por uso de drogas e tóxicos, dessa forma, a entomotoxicologia constitui campo que vem crescendo gradativamente na área da entomologia forense, ajudando, cada vez mais, devido a suas técnicas, a ter maior precisão durante a perícia em casos de overdose de drogas e medicamentos ou até mesmo por intoxicação. Porém, fatores como a bioacumulação podem interferir nesse processo, sendo necessário mais estudo nessa área com diversas espécies para ter um melhor conhecimento sobre esse fator que influencia no IPM.

Outro fator que influência muito no desenvolvimento desses insetos é a mudança de temperatura, pois, em ambientes frios, quentes, úmidos ou secos podem afetar o tempo que o inseto leva para achar o cadáver, devido aos eventos cadavéricos que acontecem nestes, após a morte, que simbolizam cada etapa de colonização desses artrópodes. Por isso, pesquisas em todo o mundo, em diversas regiões com temperaturas variadas têm sido feitas para entender melhor esses efeitos e os possíveis colonizadores de cada lugar, a fim de estimar o intervalo pós-morte com mais precisão.

No Brasil a entomologia e entomotoxicologia forense não são muito estudadas e tão pouco utilizadas nas perícias. Entretanto, é possível desenvolver técnicas em laboratórios utilizando animais, principalmente porcos, pois são os mais parecidos com seres humanos.

Contudo são necessárias mais pesquisas no campo da entomotoxicologia forense para resultados mais específicos e aprofundar esses estudos na interferência do Intervalo Pós-Morte (IPM).

\section{Referências}

ALVES, S. R. Toxicologia forense e saúde pública: desenvolvimento e avaliação de um sistema de informações como potencial ferramenta para a vigilância e monitoramento de agravos decorrentes da utilização de substâncias químicas. 2005. 132f. Tese (Doutorado) - Fiocruz, Rio de Janeiro, 2005.

AMENDT, J. et al. Best practice in forensic entomology: standards and guidelines. International Journal of Legal Medicine, Heidelberg, v. 121, n. 2, p. 90-104, mar. 2006. doi: 10.1007/s00414-006-0086-x

AMENDT, J. et al. Forensic entomology: applications and limitations. Forensic Science, Medicine and Pathology, Totowa, v. 7, n. 4, p. 379-392, dec. 2011. doi: 10.1007/ s12024-010-9209-2

AMENDT, J.; KRETTEK, R.; ZEHNER, R. Forensic entomology. Naturwissenschaften, Berlin, v. 91, n. 2, p. 5165, fev. 2004. doi: 10.1007/s00114-003-0493-5

AMENDT, J.; ZEHNER, R.; RECKEL, F. The nocturnal oviposition behavior of blowflies (Diptera: Calliphoridae) in Central Europe and its forensic implications. Forensic Science International, Limerick, v. 175, n. 1, p. 61-64, fev. 2008. doi: 10.1016/j.forsciint.2007.05.010

ANDERSON, G. S. Determining time of death using blow fly eggs in the early postmortem interval. International Journal of Legal Medicine, Heidelberg, v. 118, n. 4, p. 240-241, Aug. 2004. doi: 10.1007/s00414-004-0443-6

ANDERSON, G. S. Succession on carrion and its relationship to determining time of death. In: BYRD, J. H.; CASTNER, J. L. (Ed.). Forensic entomology: the utility of arthropods in legal investigations. Boca Raton: CRC, 2001. p. 143-175.

ANDERSON, G. S. Wildlife forensic entomology: determining time of death in two illegally killed black bear cubs. Journal of Forensic Sciences, Malden, v. 44, n. 4, p. 856-859, jul. 1999.

BACHMANN, J.; SIMMONS, T. The influence of preburial insect access on the decomposition rate. Journal of Forensic Sciences, Malden, v. 55, n. 4, p. 893-900, Jul. 2010. doi: 10.1111/j.1556-4029.2010.01403.x 
BENECKE, M.; LESSIG, R. Child neglect and forensic entomology. Forensic Science International, Limerick, v. 120, n. 1-2, p. 155-159, Aug. 2001. doi: 10.1016/S03790738(01)00424-8

BEYER, J. C. et al. Drug identification through analysis of maggots. Journal of Forensic Sciences, Malden, v. 25, n. 2, p. 411-412, May. 1980.

BOUREL, B. et al. Effects of morphine in decomposing bodies on the development of Lucilia sericata (Diptera: Calliphoridae). Journal of Forensic Sciences, Malden, v. 44, n. 2, p. 354-358, Apr. 1999.

BYRD, J. H.; CASTNER, J. L. Insects of forensic importance. In: BYRD, J. H; CASTNER, J. L. Forensic Entomology: the utility of arthropods in legal investigations. USA: CRC Press, 2001. p. 43-80.

CAMPOBASSO, C. P. et al. Drug analysis in blowfly larvae and in human tissues: a comparative study. International Journal of Legal Medicine, Heidelberg, v. 118, n. 4, p. 210-214, aug. 2004. doi: 10.1007/s00414-004-0448-1

CAMPOBASSO, C. P.; DI VELLA, G.; INTRONA, F. Factors affecting decomposition and Diptera colonization. Forensic Science International, Limerick, v. 120, n. 1-2, p.18-27, Aug. 2001. doi: 10.1016/S0379-0738(01)00411-X

CARVALHO, L. M. L. Toxicology and forensic entomology. In: AMENDT, J. et al. (Ed.). Current Concepts in Forensic Entomology. Netherlands: Springer, 2010. p. 163-178.

CARVALHO, M. L. et al. Observations on the succession patterns of necrophagous insects onto a pig carcass in an urban area of Southeastern Brazil. Anil Aggrawal's Internet Journal of Forensic Medicine and Toxicology, New Delhi, v. 5, n. 1, p. 33-39, Jan./June 2004.

CATTS, E. P. Problems in estimating the post-mortem interval in death investigations. Journal of Agricultural Entomology, Clemson, v. 9, n. 4, p. 245-255, Oct. 1992.

CATTS, E. P.; GOFF, M. L. Forensic entomology in criminal investigations. Annual Review of Entomology, Palo Alto, v. 37, p. 253-272, Jan. 1992. doi: 10.1146/annurev. en.37.010192.001345
CENTEIO, N. A. G. Entomologia forense da vida selvagem: morfologia comparada de estádios imaturos e adultos de Calliphoridae recolhidos em cadáveres de fauna selvagem. 2011. 63f. Dissertação (Mestrado) - Universidade de Lisboa, Lisboa, 2011.

CHARABIDZE, D. et al. Forenseek, un programme de simulation dédie' à l'entomologie médico-légale. Journal de Médecine Légale Droit Médical, France, v. 51, n. 3, p. 131-140, janv. 2009.

DACKO, N. B. S. The effect of arsenic trioxide on the grey flesh fly Sarcophaga bullata (Diptera: Sarcophagidae). 2011. 86f. Thesis (Master of Science) - Texas Tech University, Texas, 2011.

DEFINIS-GOJANOVIC, M. et al. Drug analysis in necrophageous flies and human tissues. Archives of Industrial Hygiene and Toxicology, Zagreb, v. 58, n. 3, 313316, Sep. 2007. doi: 10.2478/v10004-007-0022-6

ERZINCLIOGLU, Y. Z. Blowflies. Great Britain: Richmond Publishing Co., 1996.

ERZINCLIOGLU, Y. Z. The application of entomology to forensic medicine. Medicine, Science and the Law, London, v. 23, n. 1, p. 57-63, Jan. 1983. doi: $10.1177 / 002580248302300110$

FARIA, L. D. B. et al. Larval predation by Chrysomya albiceps on Cochliomyia macellaria, Chrysomya megacephala and Chrysomya putoria. Entomologia Experimentalis et Applicata, Oxford, v. 90, n. 2, p. 149-155, Feb. 1999. doi: 10.1046/j.1570-7458.1999.00433.x

FERRARI, A. C. et al. Efeito da testosterona no desenvolvimento de Chrysomya albiceps (Wiedemann) (Diptera: Calliphoridae). Medicina, Ribeirão Preto, v. 41, n. 1, p. 30-34, jan./mar. 2008.

GAGLIANO-CANDELA, R.; AVENTAGGIATO, L. The detection of toxic substances in entomological specimens. International Journal of Legal Medicine, Heidelberg, v. 114, n. 4-5, p. 197-203, Apr. 2001. doi: 10.1007/ s004140000181

GENNARD, D. E. Forensic entomology: an introduction. Lincoln: John Wiley \& Sons. Lincoln, 2007. 
GOFF, M. L. A fly for the prosecussion: how insect evidence helps solve crimes. London: Harvard University Press, 2001.

GOFF, M. L.; LORD, W. L. Entomotoxicology: insects as toxicological indicators and the impact of drugs and toxins on insect development. In: BYRD, J. H.; CASTER, J. L. (Ed). Forensic entomology. Boca Raton: CRC Press, 2001. p 331-340.

GOSSELIN, M. et al. Entomotoxicology, experimental set-up and interpretation for forensic toxicologists. Forensic Science International, Limerick, v. 208, n. 1-3, p. 1-9, may 2011. doi: 10.1016/j.forsciint.2010.12.015

GOSSELIN, M. et al. Quantification of methadone and its metabolite 2-ethylidene-1, 5-dimethyl-3,3-diphenylpyrrolidine in third instar larvae of Lucilia sericata (Diptera: Calliphoridae) using liquid chromatography-tandem mass spectrometry. Journal of Analytical Toxicology, Oxford, v. 34, n. 7, p. 374-380, Set. 2010. doi: 10.1093/ jat/34.7.374

GREENBERG, B.; KUNICH, J. C. Entomology and the law: flies as forensic indicators. Cambridge: Cambridge University Press, 2002.

GRUNER, S. V.; SLONE, D. H.; CAPINERA, J. L. Forensically important Calliphoridae (Diptera) associated with pig carrion in north-central Florida. Journal of Medical Entomology, Oxford, v. 44, n. 3, p. 509-515, May. 2007. doi: 10.1603/0022-2585(2007)44[509:FICDAW]2.0.CO;2

GUNN, J. A. et al. The determination of morphine in the larvae of Calliphora stygia using flow injection analysis and HPLC with chemiluminescence detection. Journal of Analytical Toxicology, Oxford, v. 30, n. 8, p. 519-523, Oct. 2006. doi: 10.1093/jat/30.8.519

INTRONA, F. et al. Opiate analysis in cadaveric blowfly larvae as an indicator of narcotic intoxication. Journal of Forensic Sciences, Malden, v. 35, n. 1, p. 118-122, Jan. 1990.

INTRONA, F; CAMPOBASSO, C. P.; GOFF, M. L. Entomotoxicology. Forensic Science International, Limerick, v. 120 , n. $1-2$, p. $42-47$, Aug. 2001. doi: 10.1016/S03790738(01)00418-2
KELLY, J. A. The influence of clothing, wrapping and physical trauma on carcass decomposition and arthropod succession in central South Africa. 2006. 217f. Thesis (Ph.D.) - Zoology and Entomology at University of the Free State, South Africa, 2006.

KINTZ, P. et al. Fly larvae and their relevance to forensic toxicology. The American Journal of Forensic Medicine and Pathology, Hagerstown, v. 11, n. 1, p. 63-65, Mar. 1990. doi: 10.1097/00000433-199003000-00008

KLAASSEN, C. D.; AMDUR, M. O.; DOULL, J. Casarett and Doull's toxicology: the basic science of poisons. 5 . ed. New York: McGraw-Hill, 1996.

LEFEBVRE, L.; GAUDRY, E. Forensic entomology: a new hypothesis for the chronological succession pattern of necrophageous insect on human corpses. International Journal of Entomology, Honolulu, v. 45, n. 3, p. 377-392, Jul./Sep. 2009. doi: 10.1080/00379271.2009.10697622

LIMA, C. G. P. Detecção e estudo sobre o efeito da metanfetamina e do ecstasy no desenvolvimento de imaturos de três espécies de Chrysomya (Diptera: Calliphoridae) de importância forense. 2009. 68f. Dissertação (Mestrado) - Programa de Pós-Graduação em Biologia Geral e Aplicada, Universidade Estadual Paulista Júlio de Mesquita Filho, Botucatu, 2009.

LORD, W. D. Case histories of the use of insects in investigations. In: CATTS, P. E.; HASKELL, N. H. (Ed). Entomology and death: a procedural guide. Clemson: Joyce's Print Shop, 1990. p. 9-37.

LORD, W. D.; STEVENSON, J. R. Directory of forensic entomologists. Washington DC: Water Reed Army Medical Center, 1986.

MAHAT, N. A.; ZAFARINA, Z.; JAYAPRAKASH, P. T. Influence of rain and malathion on the oviposition and development of blowflies (Diptera: Calliphoridae) infesting rabbit carcasses in Kelantan, Malaysia. Forensic Science International, Limerick, v. 192, n. 1-3, p. 19-28, Nov. 2009. doi: 10.1016/j.forsciint.2009.07.008

MILLER, M. L. et al. Isolation of amitriptyline and nortriptyline from fly puparia (Phoridae) and beetle exuviae (Dermestidae) with mummified human remains. Journal of Forensic Sciences, Malden, v. 39, n. 5, p. 1305-1313, Sep. 1994. 
MURTHY, C. R. V.; MOHANTY, M. Entomotoxicology: a review. Journal of Indian Academy of Forensic Medicine, New Delhi, v. 32, n. 1, p. 82-84, Jan./Mar. 2010.

NUORTEVA, P. Sarcosaprophagous insects as forensic indicators. In: TEDESHI, G. C.; ECKERT, W. G.; TEDESCHI, L. G. (Ed). Forensic medicine: a study in trauma and environmental hazards. Philadelphia: Saunders, 1977. v. 2. p. 1072-1095.

OLIVEIRA-COSTA, J. Entomologia forense: quando os insetos são os vestígios. 3. ed. Campinas: Millennium, 2003.

PARRY, S. et al. Accumulation and excretion of morphine by Calliphora stygia, an australian blow fly species of forensic importance. Journal of Insect Physiology, Oxford, v. 57, n. 1, p. 62-73, Jan. 2011. doi: 10.1016/j.jinsphys.2010.09.005

PAYNE, J. A. A summer carrion study of the baby pig Sus Scrofa Linnaeus. Ecology, Washington, v. 46, n. 5, p. 592602, Sep. 1965. doi: 10.2307/1934999

PAYNE-JAME, J. et al. Encyclopedia of forensic medicine and legal medicine. New York: Elsevier, 2005.

PINHEIRO, D. S. et al. Variáveis na estimativa do intervalo pós-morte por métodos de entomologia forense. Enciclopédia Biosfera, Goiânia, v. 8, n. 14, p. 1442-1458, jan./dez. 2012.

PUTMAN, J. Carrion and dung: the decomposition of animal wastes. Southampton: The Camelot Press Ltd, 1983. (Studies in Biology, 156).

ROETERDINK, E. M.; DADOUR, I. R.; WATLING, R. J. Extraction of gunshot residues from the larvae of the forensically important blowfly Calliphora dubia (Macquart) (Diptera: Calliphoridae). International Journal of Legal Medicine, Heidelberg, v. 118, n. 2, p. 63-70, Apr. 2004. doi: 10.1007/s00414-003-0408-1

SADLER, D. W. Drug accumulation and elimination in Calliphora vicina larvae. Forensic Science International, Limerick, v. 71, n. 3, p. 191-197, Feb. 1995. doi: 10.1016/0379-0738(94)01663-1
SMITH, K. G. V. A manual of forensic entomology. Oxford: Cornell University Press, 1986.

SOTO, D. A. E. Avaliação da taxa de desenvolvimento de três espécies califorídeos (Díptera) de importância forense sob o efeito de dois barbitúricos. 2008. 82f. Tese (Doutorado) - Programa de Pós-Graduação em Biologia Animal da Universidade Estadual de Campinas-Unicamp, Campinas, 2008.

SUNG, T.; MCKNIGHT, B. E. The washing away of wrongs: forensic medicine in thirteenth-century China. Michigan: University of Michigan, 1981.

TRACQUI, A. et al. Entomotoxicology for the forensic toxicologist: much ado about nothing? International Journal of Legal Medicine, Heidelberg, v. 118, n. 4, p. 194-196, Aug. 2004. doi: 10.1007/s00414-004-0442-7

VOSS, S. C.; COOK, D. F; DADOUR, I. R. Decomposition and insect succession of clothed and unclothed carcasses in Western Australia. Forensic Science International, Limerick, v. 211, n. 1-3, p. 67-75, Sep. 2011. doi: 10.1016/j.forsciint.2011.04.018

WALL R.; WARNES, M. L. Responses of the sheep blowfly Lucilia sericata to carrion odour and carbon dioxide. Entomologia Experimentalis et Applicata, Oxford, v. 73, n. 3, p. 239-246, Dec. 1994. doi: 10.1111/j.15707458.1994.tb01861.x

WATSON, E. J.; CARLTON, C. E. Insect succession and decomposition of wildlife carcasses during fall and winter in Louisiana. Journal of Medical Entomology, Oxford, v. 42, n. 2, p. 193-203, Mar. 2005. doi: 10.1603/0022-2585(2005)042[0193:ISADOW]2.0.CO;2

WATSON, E. J.; CARLTON, C. E. Spring succession of necrophilous insects on wildlife carcasses in Louisiana. Journal of Medical Entomology, Oxford, v. 40, n. 3, p. 338-347, May 2003. doi: 10.1603/0022-2585-40.3.338

WOLFF, M. et al. Detection of parathion (O,Odiethyl O-(4-nitrophenyl) phosphorothioate) by HPLC in insects of forensic importance in Medellin, Colombia. Anil Aggrawal's Internet Journal of Forensic Medicine and Toxicology, New Delhi, v. 5, n. 1, p. 6-11, Jan./Jun. 2004. 
WYMAN, J. F. et al. The temporal fate of drugs in decomposing porcine tissue. Journal of Forensic Sciences, Malden, v. 56, n. 3, p. 694-699, May. 2011. doi: 10.1111/j.15564029.2011.01725.x. 